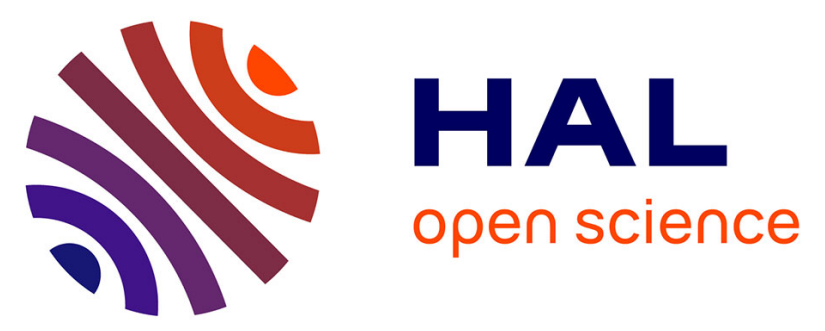

\title{
Modeling and Simulation of A Swarm Robot Application Using MBSE Method and Multi-Agent Technology: Monitoring Oil Spills
}

Khalil Aloui, Moncef Hammadi, Amir Guizani, Thierry Soriano, Mohamed Haddar

\section{To cite this version:}

Khalil Aloui, Moncef Hammadi, Amir Guizani, Thierry Soriano, Mohamed Haddar. Modeling and Simulation of A Swarm Robot Application Using MBSE Method and Multi-Agent Technology: Monitoring Oil Spills. Applied Condition Monitoring, 20, pp.96-105, 2022, 10.1007/978-3-030-85584-0_10 . hal-03183415

\section{HAL Id: hal-03183415 \\ https://hal.science/hal-03183415}

Submitted on 27 Mar 2021

HAL is a multi-disciplinary open access archive for the deposit and dissemination of scientific research documents, whether they are published or not. The documents may come from teaching and research institutions in France or abroad, or from public or private research centers.
L'archive ouverte pluridisciplinaire HAL, est destinée au dépôt et à la diffusion de documents scientifiques de niveau recherche, publiés ou non, émanant des établissements d'enseignement et de recherche français ou étrangers, des laboratoires publics ou privés. 


\title{
Modeling and Simulation of A Swarm Robot Application Using MBSE Method and Multi- Agent Technology: Monitoring Oil Spills
}

\author{
Khalil Aloui ${ }^{1,2}$, Moncef Hammadi ${ }^{1}$, Amir Guizani ${ }^{2}$, Thierry Soriano ${ }^{1}$, \\ Mohamed Haddar ${ }^{2}$ \\ ${ }^{1}$ QUARTZ Lab EA7393 - SUPMECA - 3 rue Fernand Hainaut 93400 Saint-Ouen, France \\ alouika95@gmail.com,moncef.hammadi@supmeca.fr, thierry.soriano@supmeca.fr \\ ${ }^{2}$ LA2MP, University of Sfax, Ecole National d'Ingénieurs, Sfax 3038, Tunisia \\ amir.guizani@live.fr, mohamed.haddar@enis.rnu.tn
}

\begin{abstract}
Swarm Robotics is a new approach to the coordination of a large number of robots inspired by nature. This approach aims to design collective behaviors for a large number of robots. Unfortunately, several researchers have tried to develop structured design methods but these methods are still limited. Today, swarm robotics are used in many fields, they are used in agriculture, medicine, industrial, etc. One of the most important fields that require swarm robots is surveillance. In this paper, we present in the first section some methods of designing swarm robot systems by identifying swarm engineering based on a model (MBSE) and multi-agent simulation. Then, we study the energy problem of these robotic systems and the solution proposed by the researchers. In the second section, we present an application for detecting oil in the sea and cleaning it using swarm robots. We will model this application using the MBSE method with the SysML language. Finally, we will simulate the model on a multi-agent tool to identify the functional and structural architecture of the system.
\end{abstract}

Keywords: Swarm robotic, Modeling, MBSE method, Simulation, Multi-agent technology, Sustainable energy.

\section{Introduction}

Swarm robotics is a new approach to coordinate a group of robots inspired by social insects (Sachin, 2004). It aims to design a scalable, robust and flexible collective behaviors for the coordination of a large group of robots. This approach is often inspired from social animals such as bees, ants, fish and birds (Dorigo and Birattari, 2007). Indeed, swarm robotics tends to develop a robotics system that are similar to behaviors of social animals. In particular, swarm robotics systems are meant to be robust, scalable and flexible (Camazine et al. 2003). Scalability can be defined as the ability to work with different group sizes and generate solutions for different tasks. In swarm robotics, scalability is promoted by local detection and communication. Robustness can be defined as the ability to operate despite failures in individuals. In swarm robotics, robustness is the redundancy 
and the absence of a leader. Flexibility can be defined as the ability to operate under a wide range of different environments and tasks. The simplicity of behaviors and mechanisms represent flexibility in swarm robotics.

A swarm robotic system is characterized by the autonomy of its members. These robots must be homogeneous. In some cases, there may be different types of robots in the swarm, but these groups should not be too large. In addition, the number of robots in the swarm must be large. These robots must have local communication and detection capabilities. Ultimately, robots must collaborate to achieve or improve the performance of a primary task that cannot be solved by a single robot since the detection range of a group of robots is wider than that of a single robot. (Navarro et al, 2013). Groups of robots can make decomposable tasks more efficient when they use parallelism. Moreover, a single robot is unable to operate in different places at the same time while a group of robots can do that. Another advantage is fault tolerance (i.e. thanks to the redundancy of the system, the task remains accomplished despite the failure of a single robot within a group.) In addition, swarm robot systems have several drawbacks, such as uncertainty (i.e., robots can compete rather than cooperate if they do not know what other robots are doing). Due to collisions, robots can interfere with each other in a group. Finally, the use of a group of robots in a swarm can increase the overall cost of the system (Ronald Arkin et al, 1998).

Several real-world issues require the application of swarm robots, such as monitoring an environmental accident. A swarm robot system can instantly monitor and detect hazardous events, such as a chemical spill due to its distributed detection capability. A swarm robot system can focus on one problem. First, the swarm identifies the nature of the problem. It mobilizes its members towards the source of the problem. Second, the swarm forms a patch that blocks the leak using its self-assembly characteristic (Sachin, 2004).

Depending on the given mission, the swarm robot systems can increase or decrease their number of individual robots. For example, during an oil spill, a swarm robot system will be built to contain an initial spill in the area. We will study this case in the rest of this paper.

\section{Design methods}

The design of the swarm robot system means the planning and development of a swarm system from the initial specifications and requirements. Unfortunately, there is no precise and structured methodology for designing desired collective behavior through individual behaviors. The main ingredient in developing these types of systems is always the designer's intuition.

Traditionally, many designers have used the code-and-fix approach to develop robot swarms. It is a bottom-up method means that the developer creates and improves the individual behaviors of the robots until the desired collective behavior is achieved. (Kazadi et al, 2007) applied the swarm engineering methodology called the "Hamiltonian method" to the problem of artificial physics. 
This methodology is used to create swarms of predefined global properties. They apply this methodology to the problem of artificial physics which locally creates hexagonal grids of agents. (Brambilla et al, 2014) presented a new top-down design method for swarms of robots based on prescriptive modeling and model verification called "Property Driven Design". This method consists of four phases: first, the designer specifies the requirements of the robot swarm. Second, the designer creates a normative model of the swarm. He uses model verification to verify that this normative model satisfies the desired properties; third, the designer simulates the model and implement the simulated version to validate the developed model; fourth, the designer implements the desired robot swarm to validate the previous steps. (Aloui et al. 2020) improved the "Property Driven Design" design method. They used the SysML language for the specification and modeling of the Swarm robotic system. They used SysML state machine diagrams to describe robot behaviors. Then, the robot behavior models described with SysML are implemented with a multi-agent technique (Guizani et al, 2017).

\subsection{Model Based Swarm Engineering MBSE}

In general, the model is a simplified version of a concept, relation or phenomenon to facilitate understanding of a structure or system. In the field of swarm robotics, it is possible to use the MBSE method (Model Based Systems Engineering) to model swarm robot systems to understand behavioral analysis, performance analysis, requirements traceability, system architecture, simulation, testing, etc. (Mhenni et el, 2014). The main advantage of a model-based approach is to determine the scope of the problem. That is, the developed robot swarm best meets expectations when the properties are clear and complete with the models. The detailed description of the requirements by the models reduces the risk of developing "the wrong robot swarm". To ensure model-based swarm engineering, the swarm developer can use the graphical modeling language SysML. SysML is a visual modeling language that supports the specification, design, analysis, verification, and validation of complex systems. SysML is used with different methodologies including object orientation, structured analysis, and others (Hause et al, 2006). This language includes diagrams that can be used to specify system requirements, system behavior, system structure, and parametric relationships.

\subsection{Multi-agent simulation MAS}

Recently, several researchers have started to use multi-agent systems in different fields, such as automobile design (Guizani et al, 2014a), power electronics (Hammadi et al. 2011) and aerospace design. A multi-agent system is a field of artificial intelligence (AI). (Guizani et al, 2014b) are focused on the agent and his autonomy. They defined the agent as a computer system located in its environment and able to act autonomously in order to achieve its design goals. The study of swarm robotic systems has been considered today as a study of multi-agent 
systems. Developers of swarm robot systems use realistic simulators to test and accelerate the development of new design methods. They use simulators to model the interactions between robots and the interactions of robots with their environment (Sahin et al, 2008). Luke designed a multi-agent simulation toolkit called MASON to present a large number of multi-agent simulation tasks ranging from machine learning and swarm robotics to environments of social complexity (Luke et al, 2005). Anylogic is another multi-agent simulation tool developed by The AnyLogic Company. It is a graphical modeling language that makes it easy to extend the simulation model with Java code (Borshchev et al, 2002).

\subsection{Sustainable Energy Efficiency of Swarm Robots}

Each time the groups of robots in the swarm recharge to their maximum level. Swarm robot systems do not estimate the energy needed for their next task. They require a method to minimize the overall energy cost in a swarm and simultaneously maximize the performance of the swarm. However, only a few articles in the current literature consider energy efficiency in a swarm. (Labella et al, 2004) have developed an adaptation method which manages the number of robots foraging in the environment. Indeed, they efficiently use energy when needed, but they measure only the time needed to complete the mission without measuring the amount of energy already allocated. (Liu et al, 2007) focused on energy efficiency. They modify the foraging time for each robot. (Stirling et al, 2010) have worked on flying swarms in indoor environments. They proposed a new approach to find energy-efficient search algorithms. They noticed that the progressive launch of the robots increases the search time and reduces the total energy consumption and the collision rate. (Chen et al, 2019) propose a method that improves the efficiency of energy management methods. This method uses upper and lower battery thresholds.

In the next section, we will model a swarm robot application for oil spill monitoring using the different SysML diagrams. Then, we will simulate this application on a multi-agent tool.

\section{Case study: Oil spill monitoring}

An oil spill in the areas has both immediate and long-term effects on the environment. The fauna of these areas can be directly exposed: either by swimming in the oil, by inhaling its toxic fumes, or by swallowing food affected by the oil. This exposure to petroleum can cause serious health problems and even lead to death. Thus, researchers are still trying to find solutions to clean up the affected environment (Sachin, 2004). We use the different SysML diagrams to model this application.

\subsection{Requirements specification}


The requirements diagram shown in Figure 1 specifies the application requirements of detecting environmental pollutants and cleaning them. Detecting environmental pollutants requires exploring the entire environment by deploying all the robots that detect and clean these pollutants. Cleaning should be quick and efficient using autonomous robots.

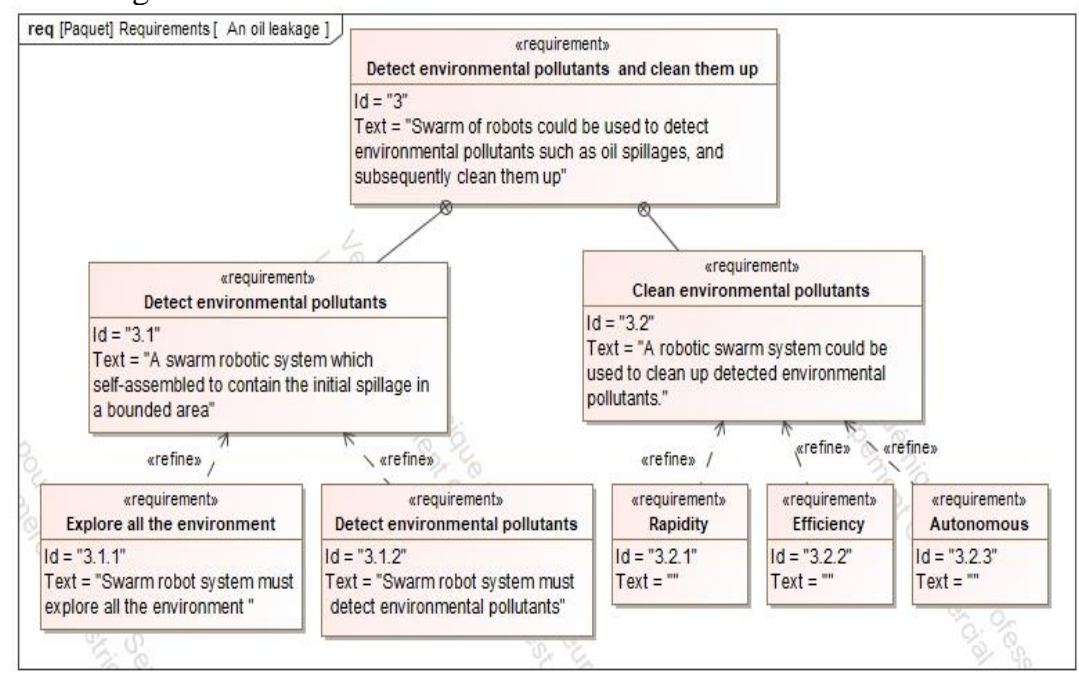

Fig. 1 Requirements diagram

\subsection{Functional architecture}

In this step, we describe the functional scenario using the different collective behaviors of swarm robots. Figure 2 shows this scenario. First, members of the swarm begin to explore the entire region. Then they detect environmental pollutants. Finally, they regroup in these areas, form groups and clean up.

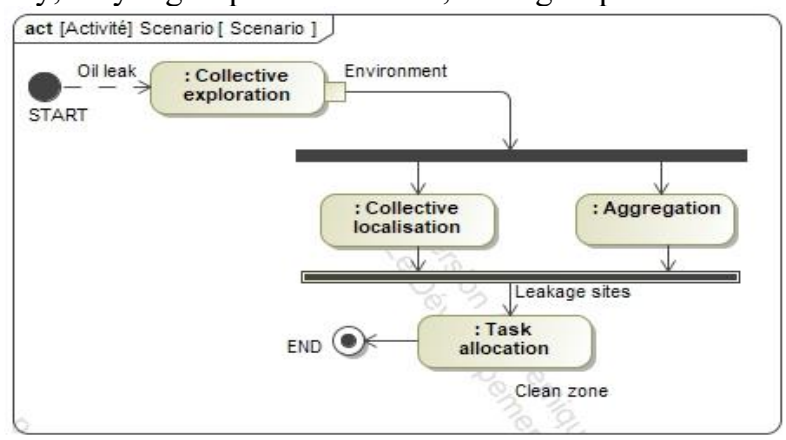

Fig. 2 Descriptive model for cleaning an oil leak

To model this case study, some collective behaviors of swarm robots are used: 
- Collective exploration: The swarm of robots cooperatively navigates the environment in order to explore and monitor it. First, the swarm is initialized with random positions and constant speeds. If the robots locate spilled oils, they send positive information to the control center. If they detect obstacles or other robots within their reach, they avoid the collision. Otherwise, they send negative information to the control center indicating that there is no oil leak in that area. Subsequently, they update their speeds.

- Collective localization: Allows the robots in the swarm to find their position and orientation in relation to each other by establishing a local coordinate system throughout the swarm.

- Aggregation: Individual robots gather spatially in a specific region of the environment. This behavior is used to ensure that the robots are assembled in the area to be cleaned.

- Task allocation: Assignment of tasks dynamically assigned emerging tasks to individual robots in the swarm. Its objective is to maximize the performance of the entire swarm system. In this case study, there are 4 groups of robots distributed throughout the area to completely clean the contaminated area.

After identifying the functional model of the system, we simulate the model using AnyLogic.

\subsection{Swarm simulation}

In this step, AnyLogic is used as a multi-agent software tool to implement the swarm simulation model. In this case study, we chose 100 robots to do this mission. As shown in Figure 4, we have divided the region into four zones according to the degree of contamination: zone A and D are the two most polluted zones. So, the swarm of robots must be directed towards them more. Zone B is the moderately polluted zone and finally, Zone E is the smallest contaminated zone. Figure 3 gives the initial number of robots in each zone.

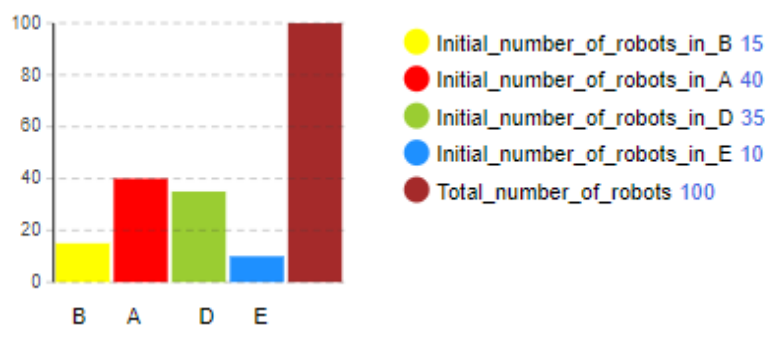

Fig. 3 Initial distribution of robots in zones

Each time, the swarm of robots must return to the boat to dispose of the captured pollutants. The initial speed of each robot is $20 \mathrm{~km} /$ hour. In fact, we have the right to direct the swarm by adjusting the probability values of the robots passing from the boat to different areas. The exploration time for the whole area is 
1 hour, the service time in each area is 50 minutes, and the time to dispose of the pollutants in the boat is 30 minutes. In addition, the robots must form swarms of 10 robots in each area. At first, robots begin to explore the entire region to identify contaminated areas. They then regroup in these areas, form groups and do the cleaning.
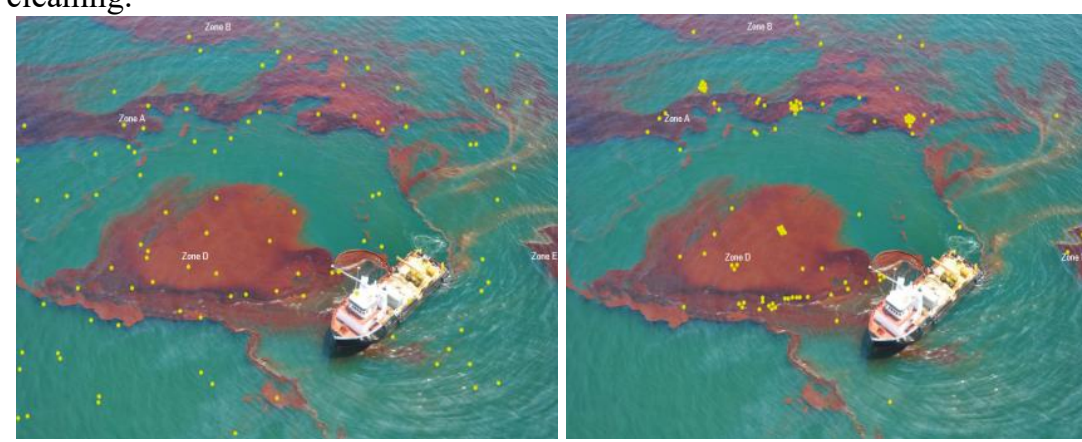

Fig. 4 Simulation of an oil spill cleanup in the ocean (a): Robots explore the entire region. (b) Robots clean the different areas

Figure 5 represents the indicators of system performance: The red curve represents the number of robots that clean area A. This number is always greater than 15 robots. Likewise, the green curve represents the number of robots that clean the area $\mathrm{D}$. This number is always greater than 10 robots. This means that the characteristics specified initially have been validated, (i.e., the robotic swarm is already directed towards the two most polluted regions). In addition, the robotic swarm treated the two less polluted areas B and E with the fewest robots. The yellow curve indicates the number of robots that return to the boat and throw the oil into the storage tanks. Because the oil diffusion is very rapid, the most important main step is to contain the spill to as small an area as possible. Each robot swarm was made up of 10 robots that scatter throughout the region and soak up the spilled oil.
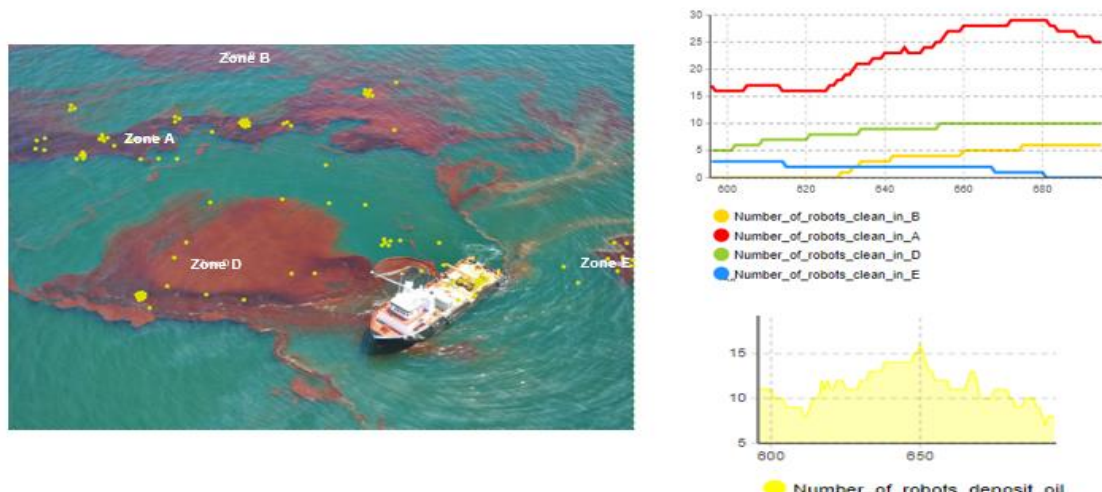

Fig. 5 System performance indicators 


\subsection{Structural architecture}

In this section, the block definition diagram shown in Figure 6 is used to define the various components of the system. The swarm consists of a group of individual autonomous robots. Several values are defined such as the size of the swarm, the area covered by the robots, the initial position and the duration of the mission.

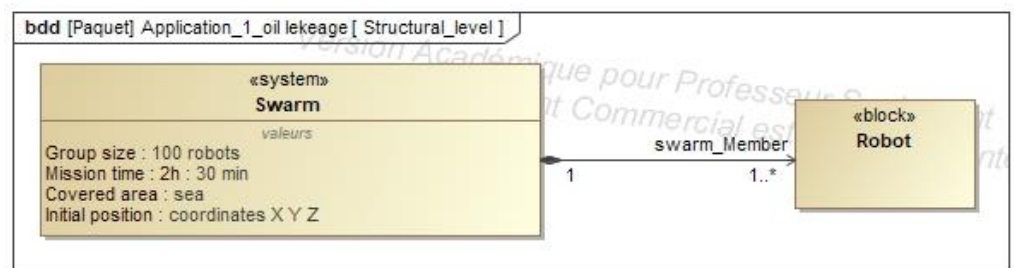

Fig. 6 The swarm architecture

In the block definition diagram shown in Figure 7 the individual robots that form the swarm is described. Indeed, each robot consists of a movement system that ensures the robot swimming in the sea, a power system, and a communication and control system. At this level, the various sensors and electronic components are defined to design the system.

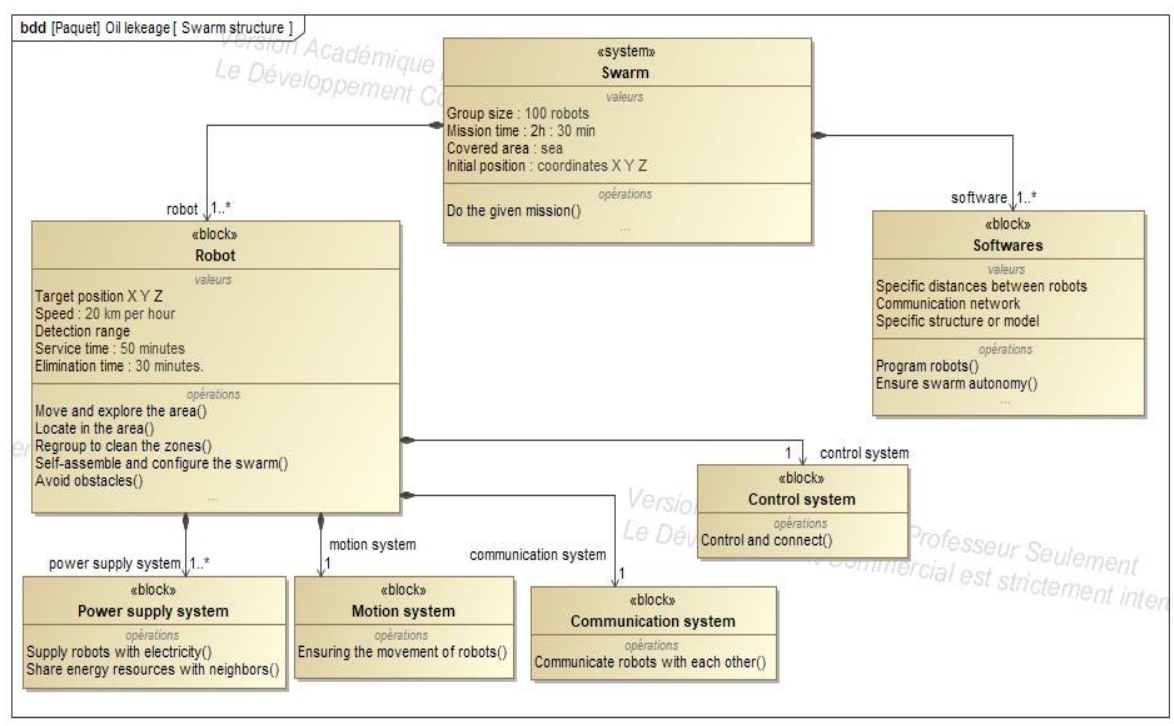

Fig. 7 Block definition diagram of a swarm of robots

Finally, it is necessary to verify that the components of the system described in the BDD correspond to the various functions of the system. The allocation matrix shown in Figure 8 relates each component of the system to its functions. 


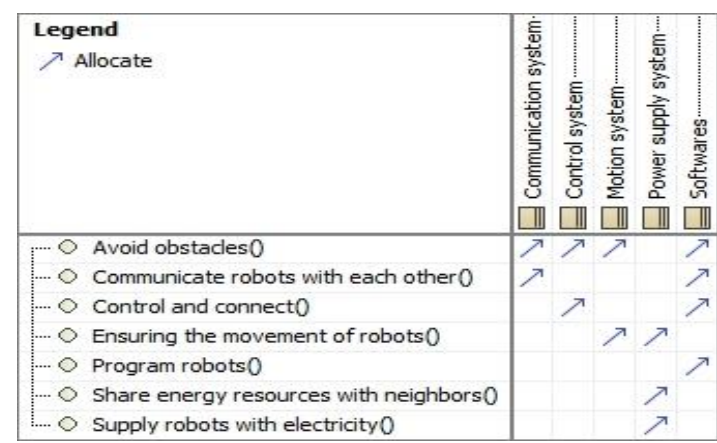

Fig. 8 Allocation matrix Component-Function

Finally, the swarm developer chooses the implantation system (like the ROS platform) to implement this system on real robots. Researchers can realize the task of implementation in further research and next steps.

\section{Conclusion}

Swarm robotics is a new approach to robot coordination inspired by social insects. This approach aims to design evolving, robust, and flexible collective behaviors for a large number of robots. Unfortunately, the design methods for these systems are still limited. Several researchers have proposed approaches to simplify design techniques, but this field is still limited.

In this paper, we have described swarm robotics: their main definition, their fields of application, their design methods and their sustainable energy efficiency. Then, we presented the modeling and simulation methods of multi-agent systems. We modeled an oil spill detection application using the MBSE method with SysML diagrams, and finally, we simulated this application on a multi-agent tool to determine the functional and structural architecture of the robot swarm. The implementation of real robots and the production of prototypes can be the subject of future papers.

\section{Références}

Aloui, K., Hammadi, M., Soriano, T., Guizani, A., \& Haddar, M. (2020). On the continuity of the swarm robot design using MBSE method and simulation. 13th International Conference on Modelling, Optimization and Simulation (MOSIM'20),

Arkin, R. C., Arkin, R. C., \& others. (1998). Behavior-based robotics. MIT press.

Borshchev, A., Karpov, Y., \& Kharitonov, V. (2002). Distributed simulation of hybrid systems with AnyLogic and HLA. Future Generation Computer Systems, 18, 829-839. 
Brambilla, M., Brutschy, A., Dorigo, M., \& Birattari, M. (2014). Property-driven design for robot swarms: A design method based on prescriptive modeling and model checking. ACM Transactions on Autonomous and Adaptive Systems (TAAS), 9, $1-28$.

Brambilla, M., Ferrante, E., Birattari, M., \& Dorigo, M. (2013). Swarm robotics: a review from the swarm engineering perspective. Swarm Intelligence, 7, 1-41.

Camazine, S., Deneubourg, J.-L., Franks, N. R., Sneyd, J., Bonabeau, E., \& Theraula, G. (2003). Self-organization in biological systems. Princeton university press.

Chen, A., Harwell, J., \& Gini, M. (2019). Maximizing Energy Battery Efficiency in Swarm Robotics. arXiv preprint arXiv:1906.01957.

Dorigo, M., Birattari, M., \& others. (2007). Swarm intelligence. Scholarpedia, 2, 1462.

Friedmann, M. (2010). Simulation of autonomous robot teams with adaptable levels of abstraction. Ph.D. dissertation, Technische Universität.

Guizani, A., Hammadi, M., Choley, J.-Y., Soriano, T., Abbes, M. S. \& Haddar, M. (2014a). Agent-Based Approach for the Optimal Design of Mechatronic Systems. Conference on Multiphysics Modelling and Simulation for Systems Design, (pp. 189-198).

Guizani, A., Hammadi, M., Choley, J.-Y., Soriano, T., Abbes, M. S., \& Haddar, M. (2014b). Agent-based approach for collaborative distributed mechatronic design. 2014 10th France-Japan/8th Europe-Asia Congress on Mecatronics (MECATRONICS2014-Tokyo), (pp. 156-161).

Guizani, A., Hammadi, M., Choley, J.-Y., Soriano, T., \& others. (2017). Multi-agent approach based on a design process for the optimization of mechatronic systems. Mechanics \& Industry, 18, 507.

Hause, M., \& others. (2006). The SysML modelling language. Fifteenth European Systems Engineering Conference, 9, pp. 1-12.

Hammadi, M., Choley, J., Penas, O., Louati, J., Rivière, A. \& Haddar, M. (2011). Layout optimization of power modules using a sequentially coupled approach. International Journal of Simulation Modelling, Danube Adria Association for Automation \& Manufacturing International Vienna, 10, (pp. 122-132 ).

Kazadi, S., Lee, J. R., \& Lee, J. (2007). Artificial physics, swarm engineering, and the hamiltonian method. World congress on engineering and computer science, (pp. 623-632).

Labella, T. H., Dorigo, M., \& Deneubourg, J.-L. (2004). Efficiency and task allocation in prey retrieval. International Workshop on Biologically Inspired Approaches to Advanced Information Technology, (pp. 274-289).

Liu, W., Winfield, A., Sa, J., Chen, J., \& Dou, L. (2006). Strategies for energy optimisation in a swarm of foraging robots. International Workshop on Swarm Robotics, (pp. 14-26).

Luke, S., Cioffi-Revilla, C., Panait, L., Sullivan, K., \& Balan, G. (2005). Mason: A multiagent simulation environment. Simulation, 81, 517-527.

Mhenni, F., Choley, J.-Y., Penas, O., Plateaux, R., \& Hammadi, M. (2014). A SysMLbased methodology for mechatronic systems architectural design. Advanced Engineering Informatics, 28, 218-231. 
Navarro, I., \& Matía, F. (2013). An introduction to swarm robotics. Isrn robotics, 2013.

Sahin, E. (2004). Swarm robotics: From sources of inspiration to domains of application. International workshop on swarm robotics, (pp. 10-20).

Şahin, E., Girgin, S., Bayindir, L., \& Turgut, A. E. (2008). Swarm robotics. Dans Swarm intelligence (pp. 87-100). Springer.

Stirling, T., Wischmann, S., \& Floreano, D. (2010). Energy-efficient indoor search by swarms of simulated flying robots without global information. Swarm Intelligence, 4, 117-143. 